\title{
Development of a 20-MeV Dielectric-Loaded Accelerator Test Facility
}

S.H. Gold, et al.

Contributed to 11th Advanced Accelerator Concepts Workshop (AAC 2004),

06/21/2004--6/26/2004, Stony Brook, New York 


\title{
Development of a $20 \mathrm{MeV}$ Dielectric-Loaded Accelerator Test Facility
}

\author{
Steven H. Gold, * Allen K. Kinkead, ${ }^{\dagger}$ Wei Gai, ${ }^{\top}$ \\ John G. Power, " Richard Konecny, " Chunguang Jing, \\ Sami G. Tantawi, ${ }^{\ddagger}$ Christopher D. Nantista, ${ }^{\ddagger}$ Y. Hu, \\ H. Chen, ${ }^{\S}$ C. Tang, ${ }^{\S}$ Y. Lin,,${ }^{\S}$ Ralph W. Bruce, ${ }^{\Delta}$ \\ Robert L. Bruce, ${ }^{\Delta}$ Arne W. Fliflet, ${ }^{*}$ and David Lewis III ${ }^{\circ}$ \\ * Plasma Physics Division, Naval Research Laboratory, Washington, DC 20375, USA \\ ${ }^{\dagger}$ LET Corporation, Washington, DC 20007, USA \\ Argonne National Laboratory, Argonne, IL 60439, USA \\ + Stanford Linear Accelerator Center, Menlo Park, CA 94025, USA \\ ${ }^{\S}$ Tsinghua University, Beijing 100084, China \\ ${ }^{\triangle}$ RWBruce Associates, Inc., Arnold, MD 21012, USA \\ - Material Science and Technology Division, Naval Research Laboratory, \\ Washington, DC 20375, USA
}

\begin{abstract}
This paper describes a joint project by the Naval Research Laboratory (NRL) and Argonne National Laboratory (ANL), in collaboration with the Stanford Linear Accelerator Center (SLAC), to develop a dielectric-loaded accelerator (DLA) test facility powered by a highpower 11.424-GHz magnicon amplifier. The magnicon can presently produce $25 \mathrm{MW}$ of output power in a 250 -ns pulse at $10 \mathrm{~Hz}$, and efforts are in progress to increase this to $50 \mathrm{MW}$. The facility will include a $5 \mathrm{MeV}$ electron injector being developed by the Accelerator Laboratory of Tsinghua University in Beijing, China. The DLA test structures are being developed by ANL, and some have undergone testing at NRL at gradients up to $\sim 8 \mathrm{MV} / \mathrm{m}$. SLAC is developing a means to combine the two magnicon output arms, and to drive an injector and accelerator with separate control of the power ratio and relative phase. RWBruce Associates, Inc., working with NRL, is developing a means to join short ceramic sections into a continuous accelerator tube by ceramic brazing using an intense millimeter-wave beam. The installation and testing of the first dielectric-loaded test accelerator, including injector, DLA structure, and spectrometer, should take place within the next year. The facility will be used for testing DLA structures using a variety of materials and configurations, and also for testing other $\mathrm{X}$-band accelerator concepts. The initial goal is to produce a compact $20 \mathrm{MeV}$ dielectric-loaded test accelerator.
\end{abstract}

\section{INTRODUCTION}

In recent years, there has been a new effort to find alternatives to the disk-loaded metal structures that are used in conventional if linear accelerators to create synchronism between the phase velocity of the wave and the particle velocity [1]. One promising concept is the dielectric-loaded accelerator (DLA), in which a smooth ceramic-lined metal tube replaces the periodic metal structure [2]. This structure can be used as a slow-wave electron accelerator by choosing a liner material with an appropriate combination of the dielectric constant and the inner and outer radii to 
match the phase velocity to $c$. The preferred material for the liner is a low-loss ceramic with a high dielectric constant. Compared to conventional iris-loaded copper slow-wave structures, the DLA geometry is simpler, potentially easier to fabricate, can have comparable shunt impedance, and permits simpler suppression of higher-order modes [3]. In addition, unlike for metal disk-loaded structures, the accelerating field is the largest field in the vacuum region of the dielectric-loaded structure. Argonne National Laboratory (ANL) and the Naval Research Laboratory (NRL) are carrying out a joint program, in collaboration with the Stanford Linear Accelerator Center (SLAC), to develop and test DLA structures for possible use in future high-gradient accelerators. ANL develops test accelerating structures, and subjects them to lowpower cold tests. The structures are then tested at the NRL Magnicon Laboratory using a high-power $11.424-\mathrm{GHz}$ magnicon amplifier tube that was jointly developed by NRL and Omega-P, Inc. in order to determine their performance at high accelerating gradients [4]. While other papers in this Proceedings present interim results from high-power tests of alumina and magnesium calcium titanate DLA structures $[5,6]$, the focus of this paper is on the overall program, whose goal is to develop a compact $20 \mathrm{MeV}$ dielectric-loaded accelerator test facility.

\section{STATUS OF THE NRL MAGNICON LABORATORY}

The heart of the NRL Magnicon Laboratory is a high-power microwave amplifier tube that was developed as an alternative to klystrons to power X-band accelerator structures. The magnicon operates over the approximate frequency range of 11.420 $11.440 \mathrm{GHz}$, and can presently produce $25 \mathrm{MW}$ of output power in 250-ns FWHM pulses at a repetition rate of up to $10 \mathrm{~Hz}$, or $10 \mathrm{MW}$ in 1.1- $\mu$ s flat-top pulses. Efforts are in progress to increase the output power to at least $50 \mathrm{MW}$ by replacing the electron beam collector, which appears to be the source of an oscillation that causes multipactor and pulse shortening in the magnicon output cavity [4]. The magnicon is a frequency-doubling amplifier that operates with a frequency-stable drive signal at $5.712 \mathrm{GHz}$ from a solid-state sweep oscillator that is monitored with a frequency counter and pulse amplified by a TWT for injection into the magnicon drive cavity. Its output is stable, even in the presence of resonant loads. The magnicon output is extracted through two SLAC-style WR-90 waveguide lines, each with a high power $\mathrm{TE}_{01}$ output window, and SLAC-style directional couplers and loads are also employed. Experiments making use of the magnicon output are connected to one of the two output waveguides, with the second terminated in a vacuum load. However, a power combiner is under development at SLAC that will permit the power from the two arms to be combined to drive a single load, or split in any desired ratio, with separate phase control, to drive separate loads, such as an electron injector and an accelerating structure. The NRL magnicon facility is illustrated in Fig. 1. Two test stands are located adjacent to the magnicon output. The first, a 5'x 25 ' raised platform for pulse compressor experiments, is 8' high, and passes over the concrete shielding wall. The second, a 10'-high concrete deck area, is currently used for testing DLA structures. A concrete bunker will be installed behind the shielding wall to provide additional radiation shielding for future accelerator experiments with an injected beam. 


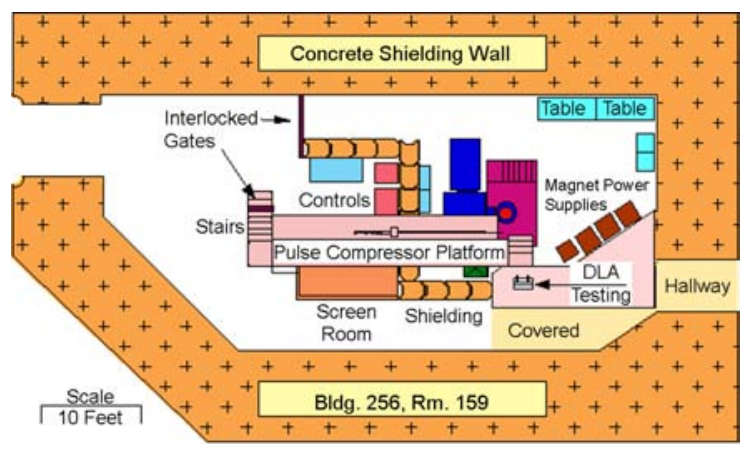

FIGURE 1. Floor plan of NRL magnicon laboratory.

\section{THE DIELECTRIC-LOADED TEST ACCELERATOR}

Figure 2 shows a schematic diagram of the complete dielectric-loaded test accelerator. A $5 \mathrm{MeV}$ injector will inject $\sim 1 \mathrm{pC}$ electron bunches into a long dielectric structure (e.g., $50 \mathrm{~cm})$. The injector and structure will be fed by separate output waveguides from a power combiner/phase shifter assembly, which will allow the injector to operate at constant power while the power and relative phase of the accelerator section is varied. The energy gain of the electron bunches will be diagnosed by a conventional magnetic spectrometer. The test accelerator will be located in a bunker behind the shielding wall in the magnicon facility. In the remainder of the paper, we describe the various separate efforts that are under way in support of this overall goal.

\section{EXPERIMENTS ON DLA STRUCTURES}

A set of experiments have been carried out on a number of traveling-wave DLA structures employing both low-loss alumina $\left(\mathrm{Al}_{2} \mathrm{O}_{3}\right)$ and magnesium calcium titanate

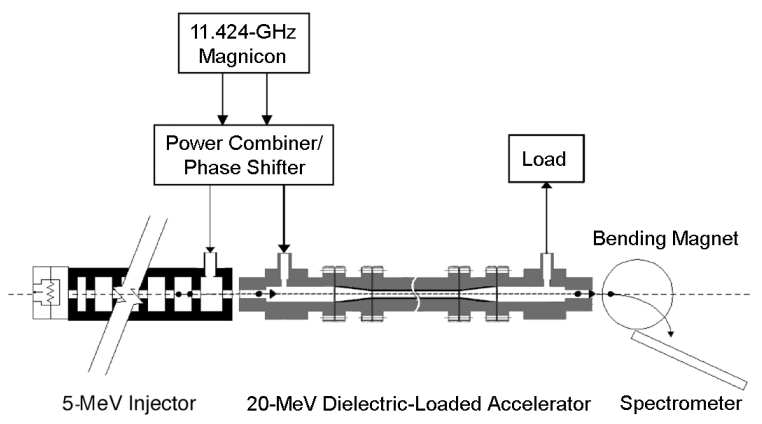

FIGURE 2. Schematic diagram of dielectric-loaded test accelerator. 


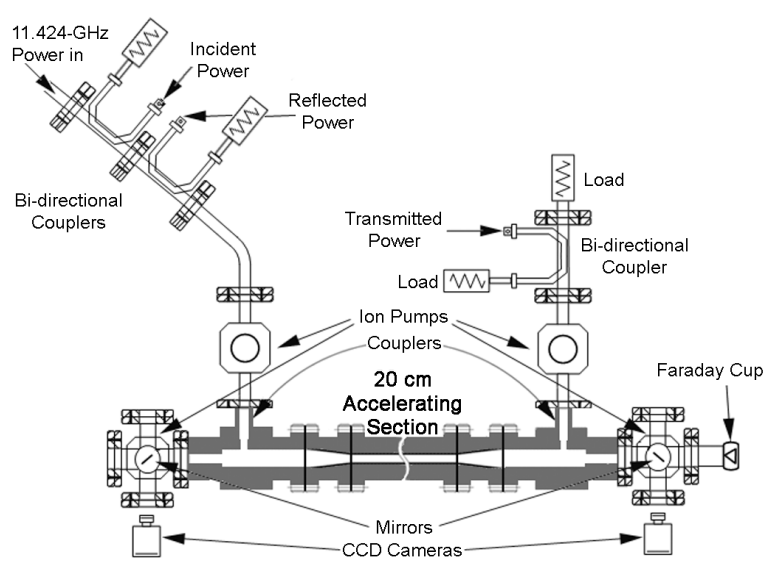

FIGURE 3. Experimental setup for DLA structure tests.

$\left(\mathrm{Mg}_{\mathrm{x}} \mathrm{Ca}_{1-\mathrm{x}} \mathrm{TiO}_{3}\right)$ ceramics. The experimental setup is illustrated in Fig. 3. The coupler design is described in detail in Ref. [7]. Measurements have been made at incident powers ranging from $10 \mathrm{~kW}$ to almost $10 \mathrm{MW}$, and accelerating gradients of up to $\sim 8$ $\mathrm{MV} / \mathrm{m}$ have been achieved. Thus far, there has been no sign of $\mathrm{rf}$ breakdown in the uniform sections of the accelerating structures. However, two key problems have been identified in these experiments: 1) Strong multipactor loading of the dielectric structures (see Ref. [8]), and 2) rf breakdown at the joints between uniform and tapered ceramic sections. Both of these problems are under experimental investigation. This work is described in more detail in two accompanying papers $[5,6]$.

\section{DEVELOPMENT OF A 5 MEV ELECTRON INJECTOR}

While accelerating gradients can be inferred from the drive power injected into the DLA structures, the real test of these structures is to use them as part of a complete accelerator configuration, in which electrons are accelerated and their energy gain directly measured. This requires an rf-driven electron injector that will produce bunched relativistic electrons for acceleration by the DLA test structures. The Accelerator Laboratory of the Engineering Department, Tsinghua University in Beijing, China, has developed a $5 \mathrm{MeV}$ electron injector that is designed to be driven by approximately $5 \mathrm{MW}$ of rf power at $11.424 \mathrm{GHz}$. The injector uses a $\mathrm{LaB}_{6}$ cathode, and a 24-cell disk and washer accelerating structure. As of the date of this Workshop, all of the injector subassemblies and focusing elements had been manufactured, and the final brazing was imminent. According to the present schedule, the injector will be ready for delivery in the Fall of 2004. Following delivery to NRL, it will be placed in a new bunker that will be installed behind the shielding wall of the Magnicon Laboratory. Figure 4 shows a schematic diagram of the injector, and its design parameters. 


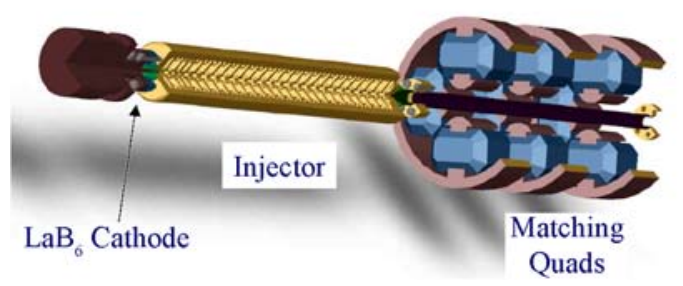

\begin{tabular}{ll|} 
Energy & $5 \mathrm{MeV}$ \\
Pulse Width & $10 \sim 100 \mathrm{~ns}$ \\
Rep Rate & $5-10 \mathrm{pps}$ \\
Charge/Bunch & $>1 \mathrm{pC}$ \\
RF Frequency & $11.424 \mathrm{GHz}$ \\
RF Power & $\sim 5 \mathrm{MW}$ \\
Phys. Emit. $(x, y)$ & $0.5 \mathrm{~mm}$ mrad \\
Energy Spread & $2-3 \%$ \\
\hline
\end{tabular}

FIGURE 4. $5 \mathrm{MeV}$ electron beam injector for DLA experiments.

\section{DEVELOPMENT OF AN X-BAND POWER COMBINER}

One essential element for this program is to develop a means to use the combined power from the two magnicon output waveguides to drive an electron beam injector and an accelerating structure. The injector will require a fixed input power, estimated to be $\sim 5 \mathrm{MW}$, while the accelerator will require variation of the input power as well as control of the relative phase of the accelerator $\mathrm{rf}$ compared to that of the electron beam produced by the injector. The two output waveguides of the magnicon have approximately equal power and a fixed phase relationship. As an important element of our collaboration, SLAC is developing a device that will combine the two magnicon outputs in a $3-\mathrm{dB}$ hybrid coupler. A second 3-dB hybrid coupler, employing a pair of movable shorts, will be used to vary the phase of one of the two inputs, in order to vary the power split in the two output arms of the first 3-dB hybrid, and a third 3-dB hybrid, also employing a pair of movable shorts, will be used to vary the relative phase of the two outputs. Figure 5 shows a block diagram of this device. The first prototype was built as a single planar monolithic device in overheight $\mathrm{TE}_{10}$ rectangular waveguide, with the phase-shifter shorts in overheight $\mathrm{TE}_{20}$ waveguide. However, it demonstrated excess loss in low power cold tests and limited power handling capability in high power tests, and will be disassembled for inspection. The final device may be built of discrete components.

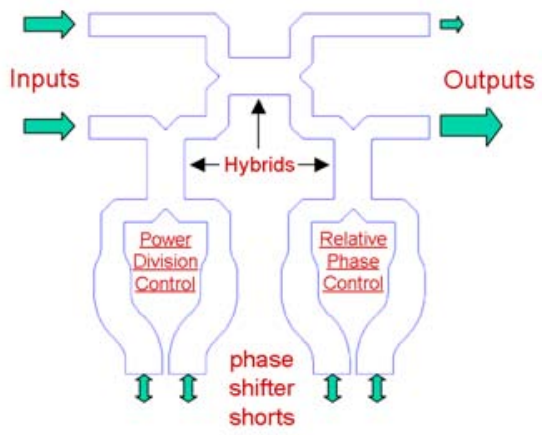

FIGURE 5. Block diagram of magnicon power combiner/phase shifter. 


\section{CERAMIC BRAZING}

One of the difficulties with the dielectric-loaded accelerator design is how to obtain suitable dielectric linings. It is currently difficult to obtain single-piece ceramic tubes of the required materials, such as high purity, high density alumina, in the required lengths (perhaps $1 \mathrm{~m}$ per accelerator section), while meeting the necessary tolerances on inside and outside diameters, roundness and straightness. The present hot-pressed high purity alumina tubes being evaluated for the DLA are only available in lengths of $\sim 10 \mathrm{~cm}$. Thus, the initial DLA experiments use mechanical assemblies of ceramic tube sections loaded into a metallic tube. This technique places stringent requirements on the tube segment end tolerances and the assembly procedures, and may cause difficulties with subsequent handling and with thermal cycling during bakeout, where the large thermal expansion mismatch between the ceramic tube segments and the metallic liner can produce gaps between the tube segments. Such gaps can cause performance degradations through impedance mismatches and localized breakdown.

RWBruce Associates, Inc., working with NRL, has been exploring the use of reactive oxide glass brazes and localized millimeter-wave beam heating of the joint region to produce joints that are mechanically strong, with uniform dielectric properties, and with negligible geometric discontinuities (see Fig. 6). The approach uses rotating fixturing, susceptors to improve the millimeter-wave coupling to the ceramic materials, and radiation shielding to ensure uniform heating of the joint region through a thermal cycle ranging from $800^{\circ} \mathrm{C}$ to $1500^{\circ} \mathrm{C}$, in order to bond the material, diffuse the braze material into the joint, and recrystallize the joint region. The millimeter-wave beam system permits use of simple fixtures for maintaining alignment, rotating samples, and applying pressure to joints, and the use of inexpensive instrumentation for process control and monitoring. The final result is a crystalline joint with dielectric, thermal and mechanical properties very similar to the material being joined. The dielectric properties have been confirmed by network analyzer measurements at ANL. The remaining work is to develop techniques for producing a series of joints in a long assembly while maintaining alignment, and for machining the inner and outer diameters of the ceramic tube assembly to meet the specifications. These problems appear readily soluble. It is thus felt that the millimeter-wave beam reactive frit brazing ap-

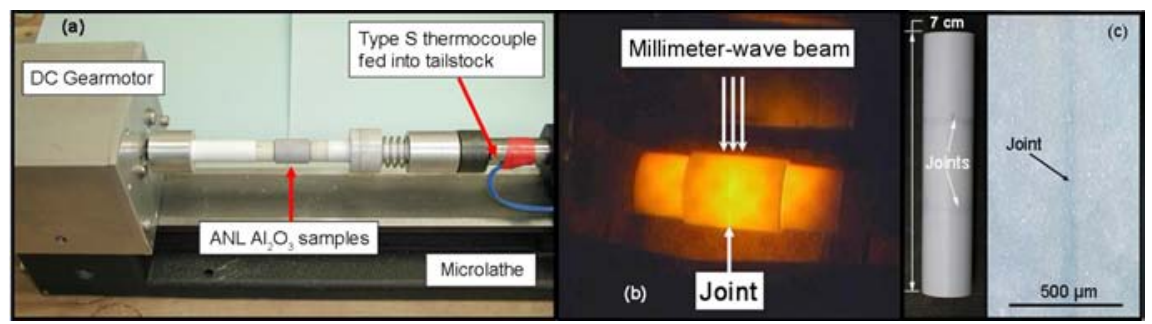

FIGURE 6. (a) View of microlathe used for sample rotation while heating with the millimeter-wave beam, with controlled pressure applied to joint via compression spring. Internal temperature measured with thermocouple in inner support tube, and external temperature measured with two-color pyrometer. (b) View of joint being heated by $83-\mathrm{GHz}$ millimeter-wave beam while being rotated. (c) Set of 3 joined tubes with micrograph showing nearly indistinguishable crystalline joint region. 
proach has an excellent chance of providing the dielectric liners required for the DLA.

\section{SUMMARY}

The goal of this project is develop a test bed to study structure-based advanced accelerator concepts in X-band, and in particular, dielectric-loaded accelerated structures, as illustrated in Fig. 3. The heart of the facility is an 11.424-GHz magnicon amplifier that can produce $25 \mathrm{MW}$ of output power, split evenly over two waveguide feeds, in 200-ns FWHM pulses. To date, only a single output arm has been used in the structure tests. Efforts are in progress to increase the available power to at least 50 MW by eliminating a collector multipactor problem that is causing pulse-shortening of the magnicon output pulse. In order to move from gradient tests of accelerating structures to actual acceleration tests, an electron injector is required. Such an injector is being built by the Tsinghua University in Beijing, China, in an agreement with ANL. It is designed to produce $\sim 1 \mathrm{pC}$ bunches of $5 \mathrm{MeV}$ electrons for injection into the accelerating structure. However, the accelerator configuration will require separate power and phase control for the injector and the accelerating structure. To permit this, a power combiner/phase shifter is being developed for the project by SLAC. A project related to the DLA structure development has been under way by RWBruce Associates, Inc., in collaboration with NRL. The goal of the project is to develop a means to braze ceramic tubes into a continuous structure, without discontinuities in the joint region that can lead to mismatches, field enhancement, and rf breakdown. This project has demonstrated the ability to achieve mechanical joints with good rf properties between high-purity alumina sections that are difficult to join by conventional means. All of these related efforts are intended to lead to a working dielectric-loaded test accelerator within approximately one year.

\section{ACKNOWLEDGMENT}

This work was supported by the Office of High Energy Physics, US Department of Energy and by the US Office of Naval Research.

\section{REFERENCES}

1. J. G. Power and W. D. Kimura, in Advanced Accelerator Concepts, edited by C. E. Clayton and P. Muggli, AIP Conference Proceedings 647, New York: AIP, 2002, pp. 156-164.

2. P. Zou et al., Rev. Sci. Instrum. 71, 2301-2304 (2000).

3. E. Chojnacki et al., J. Appl. Phys. 69, 6257-6260 (1991).

4. O. A. Nezhevenko et al., "Performance of X-Band Pulsed Magnicon Amplifier," in Proc. 2003 Particle Accelerator Conf., edited by J. Chew et al., Piscataway, NJ: IEEE, 2003, pp. 1128-1130.

5. J. G. Power et al., paper in this Proceedings.

6. C. Jing et al., paper in this Proceedings.

7. W. Liu et al., "New rf Design for $11.4 \mathrm{GHz}$ Dielectric-Loaded Accelerator," in Proc. 2003 Particle Accelerator Conf., edited by J. Chew et al., Piscataway, NJ: IEEE, 2003, pp. 1810-1812.

8. J. G. Power et al., Phys. Rev. Lett. 92, 164801 (23 April 2004). 